\title{
Minutiae-based Triangular Acreage Fingerprint Match Method
}

\author{
Gang Wang, Dan Zhao and Xiaoyu Sun \\ ${ }^{1}$ School of Renewable Energy, Shenyang Institute of Engineering, Shenyang, \\ China \\ *Corresponding author:wanggang@sie.edu.cn
}

\begin{abstract}
Fingerprint recognition systems are the top topics of biometric research and more researchers' effort is provided to improve high accuracy and speed. Matching methods based on the minutiae points are commonly used and are effective in practice. Algorithms which based on minutia triplets among them are attractive matchers. However, recognizing fingerprints in poor quality images, direction changed, size different fingerprints and so forth is still a very complex job. To overcome these drawbacks, a fingerprint match method used minutiae-based triangular acreage is described here to improve matching accuracy.
\end{abstract}

Keywords: triangular acreage, minutiae, fingerprint match

\section{Introduction}

With the development of automatic machines, self-service, bank cards and internet shopping, the demand to identify people uniquely, safely and quickly is becoming the basic factor during social life experiences. The traditional methods, such as passwords and cards, cannot be trusted to establish a person's identity due to passwords or PIN can be calculated by hacker's interruption and cards can be stolen, lost or easily duplicated. In these cases, biometric recognition becomes the most attractable solution to above problems.

Biometric traits, e.g., fingerprints, face, iris, ear, can provide unique characters of one person and cannot be easily stolen or shared compared with traditional security methods (such as key and password) [1]. Due to their better security, convenience and easily recognized with the help of modern technology, they are deployed in a large amount in our daily life and are playing more and more important role in various security applications. Biometric is accepted as an essential component of effective person identification solutions because biometric identifiers intrinsically represent the individual's identity. Among these biometric traits, fingerprint has an overall desirable property when compares with others, such as easily accessible, permanent unchangeable and good recognition accuracy, and is becoming the most popular biometric trait.

Fingerprints have been used for identifying individual from the ancient time. Historically, fingerprint recognition systems were mostly used in forensic sciences, and due to their strongpoint, such as uniqueness and permanence, fingerprint is among the most widely used biometric traits with a broad range of both government and civilian applications at present. In other words, fingerprints are a sort of identity card that people carry with them continuously. The current popularity of these systems is mainly focused on civilian applications such as building access systems, personal computer login, ATM authentication and other similar situations, company with the high complexity of fingerprint matching, time consuming and the huge amount of existing fingerprints, it is necessary to build computer systems that automatically process fingerprints with high accuracy and low needed waiting time. 
The general purposes in the automatic recognition systems include fingerprint acquisition, verification, identification and classification. Today, fingerprint recognitions are widespread accepted on the field of person identification and verification. Fingerprint recognition system is asked to accept or reject the hypothesis of matching between a stored fingerprint image and the test image. Consequently, the system must match the person's characteristics with those stored in the database as the normal. Identification requires the system match the characteristic of the person to be recognized against the whole set of characteristics stored in the database, thus deciding if one of the items of reference information is sufficiently similar to the one considered. Fingerprint matching is a difficult patternrecognition problem due to large intra class variations (variations in fingerprint images of the same finger) and large interclass similarity (similarity between fingerprint images from different fingers). That is why fingerprints from the same finger may sometimes look quite different whereas fingerprints from different fingers may appear quite similar. Generally speaking, it is difficult to match a provided fingerprint with an enrollment fingerprint from the same person due to deviations caused by factors including noise, non-linear geometric distortions and inconsistencies in fingerprint pressure to a human fingerprint expert, not the mention to these automatic machines. In fingerprint recognition field, matching methods and algorithms are attracting more attention from research area. To develop more sophisticated algorithms that improve accuracy, robustness, and efficiency in fingerprint recognition is a very active research area, and the key component in fingerprint recognition systems is the fingerprint matching algorithm.

Fingerprint-matching algorithms include minutiae-based matching [2], transform feature-based matching [3], graph -based matching [4] and hybrid feature-based matching[5]. Minutiae-based representation is storage efficient and testified to be a high efficient way during the last several hundred years. Minutiae-based fingerprintmatching is the commonly used one among these.

The most obvious structural characteristic of a fingerprint is a pattern of interleaved ridges and valleys in a fingerprint image, ridges are dark whereas valleys are bright [6]. In the biometric process of fingerprint recognition process, minutiae are specific points in a fingerprint image. There are two main types, known as ridge endings and bifurcations. Sometimes, other details, such as the points at which scars begin or terminate, are considered minutiae. Minutiae are typically represented as $(\mathrm{x} ; \mathrm{y} ; \theta)$; where $(\mathrm{x} ; \mathrm{y})$ represent the two-dimensional plane point coordinates, and $\theta$ represents the ridge direction at that point. The number and locations of the minutiae vary from finger to finger in any particular person, and from person to person for any particular finger. The number of minutiae in a fingerprint can be varied from several to more than 100 and the entirely match of a small number of minutiae, generally near fourteen is sufficient to decide that the two fingerprint originate from the same finger with high confidence. When a set of finger images is obtained from an individual, the number of minutiae is recorded for each finger. The precise locations of the minutiae are also recorded. The result is then can be entered and stored in a computer database. A computer can rapidly compare this function with that of anyone else in the world whose fingerprint has been scanned.

Minutiae-based matching means that minutiae are extracted from the normal fingerprints and stored as sets of points in the two-dimensional plane. Minutiaebased matching essentially consists of finding the alignment between the template and the input minutiae sets that result in the maximum number of minutiae pairings. Fingerprint Recognition using Minutia Score matching system.

The overall flow of the minutiae-based fingerprint matching algorithm is typically four steps. First, the algorithm computes similarity between minutiae of 
two fingerprints by comparing minutiae pairs by the essential factors which behind the minutiae. That is compared the provided one with the minutiae template, and the matched minutiae will be found. Next, it aligns two fingerprints according to the most similar minutiae pair. The algorithm then establishes minutiae correspondence-minutiae that are close enough both in location and direction are deemed to be corresponding minutiae. Iterate last steps $\mathrm{N}$ times and then return the maximum number of matched minutiae. Finally, the algorithm computes a similarity score to reflect the degree of match between two fingerprints based on factors such as the number of matching minutiae, the percentage of matching minutiae in the overlapping area of two fingerprints, and the ridge count between matching minutiae. A fingerprint match score should be high for fingerprints from the same finger and low for those from different fingers. If the matching score exceeds a predefined threshold, the two fingerprints can be regarded as belonging to the same finger.

Automatic fingerprint recognition systems offer a reliable, rapid, consistent, and cost-effective solution in a number of traditional and emerging applications that require person recognition. Although state-of-the-art automatic fingerprint systems have impressive performance, they still cannot match the performance of a fingerprint expert in handling poor quality and latent fingerprints.

We observed that there is no significant change in the acreage if the minutia appears as a ridge ending in one impression and as a bifurcation in another impression of the same finger.

This paper is organized as follows: Section 2 outlines the related work and details its components in a simple way. Section 3 provides overall system procedures and explained its merits in dealing with all kinds of uncommon situations.

\section{Related Work}

During recent decades, minutiae-based fingerprint matching method plays an important role in automatic fingerprint recognition system. For processing fingerprint images and extracting obvious features, many algorithms are developed. Minutiae matching may suffer from distortion, missed minutiae and spurious minutiae. To overcome these obstacles, several kinds of minutiae-based algorithms have been published and various kinds of algorithms have been provided to improve performance during fingerprint automatic recognition process.

To solve the alignments of regions far away from the reference minutiae, this team proposes a minutia matching method based on global alignment of multiple pairs of reference minutiae in 2005[7]. In 2005, the team proposes a fingerprint feature named the adjacent feature vector (AFV) for fingerprint matching. An AFV consists of four adjacent relative orientations and six ridge counts of a minutia. Given a fingerprint image, the optimal matching score is computed in three stages: minutiae candidate pairs searching based on AFVs; coordinate transform for image rotation and translation; and transformed minutiae matching to get matching score.[8] In 2008, a minutiae-based fingerprint matching algorithm is proposed to solve two correspondence and similarity computation problems. For the correspondence problem, he assigns each minutia two descriptors: texture-based and minutiae-based descriptors, and use an alignment-based greedy matching algorithm to establish the correspondences between minutiae. For the similarity computation, he extracts a 17-D feature vector from the matching result, and convert the feature vector into a matching score using support vector classifier [9].

Triangular matching is provided to cope with the strong deformation of fingerprint images derived from static friction or finger rolling. As to triangles related algorithms, there are below progresses. 
In 2000, this team describes a fingerprint verification algorithm based on triangular matching to overcome major distortion. In their algorithm, the test fingerprint minutiae set are warped to the locations of the template fingerprint minutiae set to cope with distortion. But the side effect is that the accumulation of local variations may also cause false matching between non-corresponding fingerprints [10].

In 2006, these researchers propose a fingerprint matching algorithm, in which the local properties of each minutiae triplet were adopted to find potential corresponding triangles and tolerate reasonable distortion, then a genetic algorithm was adopted to find the globally optimized transformation [11].

In 2009, a new minutiae template-merging approach based on hierarchical Delaunay triangulations is provided. Their key idea is synthesizing a super-template from multiple enrollment templates to increase coverage area, restore missing features, and alleviate spurious minutiae. Each minutia in the super-template is assigned a weight representing its frequency of occurrence, which serves as a minutiae quality measure. During the merging stage, they employ a hierarchical, weight based, scheme to search for a valid alignment between a given template and the super-template [12].

In 2012, this team identifies the quality parameters that have a more significant impact on fingerprint matching accuracy and creates a more accurate matcher called M3gl. M3gl arranges the minutiae in clockwise sense and the comparison function performs the three possible rotations of the triplets achieving the invariance to the order of minutiae in the feature and the sensitivity to the reflection of minutiae triplets [13].

\section{Method}

In this paper, a minutiae-based triangular acreage fingerprint match method is proposed to provide a better performance of automatic fingerprint matches. This technique is able to match fingerprints from different machines and acquired in different situations.

The basic thinking is to divide the whole fingerprint every neighbor three points and connect these three points with straight lines, then calculate the acreage of it. Minutiae are known as the typically characters of fingerprint especially when automatic recognition machines are used, thus we match fingerprints based on minutiae's relatively position will keep stable during different situations and we choose area and inner-angles simultaneously as the behalf of position to match fingerprints automatically. The goal of this paper is to study $\mathrm{t}$ he fingerprint methods and to develop a system that is used to overcome the drawbacks and increase the performance of fingerprint matching.

Compared with other traditional matching methods, this method is able to handle more complicated fingerprint images such as skew or rotation, displacement, unevenness, different background brightness and size, partial overlap, missing and spurious minutiae points. Main calculation load in this method is algebraic operation and hierarchy indexes also promote the matching speed. The execution time of the method can be reduced and the accuracy will be improved.

\subsection{Detailed Process}

When two fingerprints are being matched together and we do not intend to align them in advance, thus we need to calculate all neighbor three minutiae points of one fingerprint and then match the acquired acreage set of the other fingerprint with these acreage sets of the first fingerprint. Acreage relatively values are the main 
index during the compare and the corresponding three inner-angels are as the subsidiary index to align and for the further confirmation.

This defines some basic functions that we use throughout this paper. Given three minutiae

$$
\mathrm{M} 1=(\mathrm{x} 1 ; \mathrm{y} 1 ; \theta 1) \mathrm{M} 2=(\mathrm{x} 2 ; \mathrm{y} 2 ; \theta 2) \text { and } \mathrm{M} 3=(\mathrm{x} 3 ; \mathrm{y} 3 ; \theta 3)
$$

$\mathrm{A} 123=\mathrm{A}(\mathrm{M} 1 ; \mathrm{M} 2 ; \mathrm{M} 3)$

$$
\mathrm{R} 123=\mathrm{R}(\mathrm{M} 1, \mathrm{M} 2, \mathrm{M} 3)=\mathrm{A} 123 / \Sigma\{\mathrm{A} 123+\mathrm{A} 234+\mathrm{A} 124+\ldots \ldots\}
$$

$\theta 123=(\theta 23, \theta 13, \theta 12)$
Equation (1)

Equation (2)

Equation (3)

Equation (4)

A (M1; M2; M3) represents the triangular acreage which is composited by M1, M2 and M3 in Equation (1). R (M1, M2, M3) represents the ratio of the acreage of this triangular to the whole acreage of the fingerprint. $\theta 23$ in radian represents the inner-angle is composed by line M1M2 and M2M3, $\theta 13$ and $\theta 12$ are in similar definition. In $\theta 123$, the order of these three inner-angles is arranged randomly.

Firstly, from the boundary minutiae the every three neighbor minutiae are divided into groups and then connect these three points with straight lines to composite one triangle separately. Calculate the acreage of every triangle and record the three inner-angles, also can we catch the whole acreage of the fingerprint by simply add them together. In the case of same resolution, compare the acreage of the two fingerprints and reduce the surrounding minutiae to be the same as the smaller one. Then repeat the first step again and normalize the acreage values to be the percentage of the separated total acreage. Then we composite the acreage set in this manner:

$$
\begin{aligned}
& \text { SETA } 1=(\mathrm{Rj}, \mathrm{Ri}, \mathrm{Rk}, \ldots \ldots) \\
& \operatorname{SET} \theta 1=(\theta \mathrm{j}, \theta \mathrm{i}, \theta \mathrm{k}, \ldots \ldots)
\end{aligned}
$$$$
\text { Equation (5) }
$$

In Equation (5), the values of $\mathrm{R}$ are sorted in ascending order. In the same manner we got SETA2 of the other fingerprint. In Equation (6), the order of $\theta$ is in accordance with the related triangle in Equation (5). Through the composition of the acreage set and the correlated angles set by the ascending order and we got two pieces of sets.

Secondly, we began to compare these two sets. During the process, acreage relatively values are the main index and the corresponding three inner-angels are as the subsidiary index. For two given SETA1 and SETA2 sets, we compare them to find the most similar value and the check their corresponding three inner-angles to find the most compatible triangles first, that is, the ratio is equal or nearly equal, and this decision is made by the criteria. Of course during the above process, two different thresholds have been set in advance and can be tuned later. If the first ratio equal triangle is not similar with the corresponding one in the other set after three inner-angle match failed by a round. Then the second ratio equal triangle is provided to execute the same procedure. If no such one fitted in the equal ratio procedure, then end the match process. If ratio equal triangles can be found but their angles cannot match, the process is also ended.

If the matched triangles have been found, then with the aid of computer program, find the most matched positions triangles and the use them as the reference points to adjust the position of these two fingerprints to align them. Then common procedures are adopted to finish the whole matching process. 
From above process, we can easily draw the conclusion that compare the acreage ratio of the triangles is effective to deal with triangles mirror and displacement.

\subsection{How to Deal with Variability of the Same Finger}

3.2.1. Skew or Totation: The same finger may be skewed or rotated at different angles when contact to the surface of fingerprint scanners during different approaches. This result in finger skews up to $\pm 20^{\circ}$ (regard to vertical orientation) in extreme cases [1], and makes it difficult to compare fingerprints automatically.

As described above, the method we provide is align-free or auto aligned, and by means of the adjust process a skewed fingerprint can be easily set to the right direction with the other fingerprint no matter what offset occurred whether this is caused intentionally or unintentionally.

3.2.2. Displacement: A typical pore has a diameter of about 5 pixels $(115.5 \mu \mathrm{m})$ and the adjusted average spacing between intra-ridge pores is 13 pixels $(300.3 \mu \mathrm{m})$ [14]. The same finger may be placed on different areas of the surface of fingerprint scanners during different approaches. The displacement will be so tiny that the man will hardly feel it but on the view from pixels it will be a large number.

To traditional match algorithm, it will be a hard core to deal with. But as to the above method, it will be easily to overcome the displacement by the help of adjust and align process.

3.2.3. Unevenness: During the operating process, mainly due to factors of non-linear distortion, pressure, noise, quantization error and skin condition, the fingerprint will no longer reflect the real ratio of the relatively distance and angle of minutiae, effect expand or withdraw in some extent. Non-linear distortion reflects the inevitable affect when a three-dimensional object is projected on a two-dimensional scanner surface. And as we known, the skin of fingerprint is plastic and the surface of the fingerprint is various in different person or even different fingers from the same person. At the same time, the factor of pressure when people print their fingers is different is also to be considered. Noise and quantization error are introduced by the electronic devices interference, smear on the scanner's surface, and the inevitable error to process the real image to be a binary code set. These various situations challenge the match method especially under the automatic process.

The method it this paper is based on the proportion of acreage values. Here let us assume only one minutiae point is changed, and compare with these methods apply absolute values, the ratio of relative is smaller than the absolute value. To some extent, this method is anti-distort. This is the first reason. Then we can adjust the threshold to tolerant this kind of unevenness appearance, but to get a trade-off between accuracy and security, this criteria cannot be expand infinitely. But to the noise and quantization error, this method cannot provide better solution compare with others. From this point of view, the method here can only overcome unevenness to some extent.

3.2.4 Background Brightness and Size: As describes above, the main index is the acreage of triangles and the subsidiary index is the three inner-angles, the method provided here is not relevant with the brightness of fingers and merely relates with the position of minutiae, and is effective to compare from different fingerprint scanners and different background brightness. Also due to the introduction of compare of minutiae points at the first stage of the method, it is easily to deal with different fingerprint size during the match process. 
3.2.5. Partial Overlap: Sometimes fingerprint can be partial overlap especially serious for small-area scanners. This will result in part of the fingerprint area to fall outside the data collection area on the map and a smaller overlap. It is will be a large trouble to the automatic recognizers. To solve this, the method above must be improved to add a procedure to recognize the fingerprint part by part. This will need longer time and a heavy workload to the processor. To some partial overlap it will be effective, but to others, it will be no useful.

3.2.6. Missing and Spurious Minutiae Points: During the whole fingerprint collection process, it is easily to miss some minutiae points or add some non-exist minutiae points which are contributed the above discussed items, such as noise, quantization error and so on.

To missing minutiae points, it is manifested in the triangular acreage is larger than the original fingerprint pattern and inverse to the spurious minutiae points. In both cases, the main index and the subsidiary index will no longer match, but the whole acreage is regarded as unchanged and do not affect the ratio of other triangular, thus will not affect other minutiae point match and only affect themselves. If the threshold is not rigid, then these two kinds of cases can be neglected. If needed, can also report the ration of missing and spurious minutiae points after two fingerprints are matched finally. It is proven that this method making the whole process less sensitive to missing and spurious minutiae points.

In a word, this method provides a new thinking can be applied in the matching process.

\section{Conclusion and Future Work}

Different from most existing triangle matching algorithms, the proposed method puts more emphasis on minutiae's stable relationship by the aid of acreage in spite of the brightness of fingerprint samples and the computation of matching scores. Minutiae-based triangular acreage fingerprint matching methods have proved to be fast and overcome the relative nonlinear deformation present in the fingerprint image pairs, but effectively incorporating various features with minutiae in fingerprint matching still is a challenging task. The threshold needs to be adjusted in subtle minor to fulfill the performance to be improved for a wide adoption in many applications.

In the near future we plan to return to search more effective way of fingerprint minutiae normal protection, also perform extensive experiments and comparisons with competing approaches to demonstrate the proposed approach using all current challenging public database study the behavior of the new method for cancelable fingerprint template.

\section{Acknowledgements}

The author would like to thank the reviewer for the valuable comments and the grammar of this paper. This work is partly supported by China Scholarship Council and Shenyang Institute of Engineering.

\section{References}

[1] D. Maltoni, D. Maio, A. K. Jain, S. Prabhakar. Handbook of Fingerprint Recognition, second ed. , Springer (2009).

[2] A. K. Jain, L. Hong, R. Bolle. On-Line fingerprint verification, IEEE Trans. Pattern Anal. Machine Intell, 19, 302 (1997)

[3] A. K. Jain, S. Prabhakar, L. Hong, S. Pankanti. Filter Banker-based Fingerprint Matching. IEEE Trans. Image Process, 9, 846 (2000). 
[4] D. K. Isenor, S. G. Zaky. Fingerprint Identification Using Graph Matching. Pattern Recognition, 19, 113 (1986).

[5] A. Ross, A. Jain, Reisman. A Hybrid Fingerprint Matcher. Pattern Recognition 36 (7), 1661-1673.,

[6] D. R. Ashbaugh. Quantitative-Qualitative Friction Ridge Analysis: An Introduction to Basic and Advanced Ridgeology, CRC Press, Boca Raton, FL, (1999).

[7] E. Zhu, J. P. Yin, G. M. Zhang. Fingerprint Matching Based on Gobal Alignment of Multiple Reference Minutiae. Pattern Recognition, 38, 1685 (2005)

[8] X. F. Tong, J. H. Huang, X. L. Tang, D. M. Shi. Fingerprint Minutiae Matching Using the Adjacent Feature Vector. Pattern Recognition Letters, 26,1337 (2005).

[9] J. J. Feng. Combining Minutiae Descriptors for Fingerprint Matching, Pattern Recognition, 41,342 (2008).

[10] Z. M. Kovacs-Vajna. A Fingerprint Verification System Based Ontriangular Matching and Dynamic Time Warping. IEEE Trans. Pattern Anal. Matchine Intell, 22, 1266 (2000)

[11] X. Tan, B. Bhanu. Fingerprint Matching by Genetic Algorithms.Pattern Recognition 39, 465 (2006)

[12] U. Z. Tamer, B. George, E. Ali and P. Salil. Minutiae-Based Template Synthesis and Matching Using Hierarchical Delaunay Triangulations, Computer Vision and Image Understanding, 113, 979 (2009)

[13] Miguel Angel Medina-P'erez, Milton Garc'ia-Borroto, Andres Eduardo Gutierrez-Rodr'1guez and Leopoldo Altamirano-Robles. Improving Fingerprint Verification Using Minutiae Triplets, Sensors, 12, 3418 (2012)

[14] C. J. Lakhmi, H. Ugur, H. Isao, S. B. Lee, T. Shigeyoshi. Intelligent Biometric Techniques in Fingerprint and Face Recognition, CRC press, (1999)

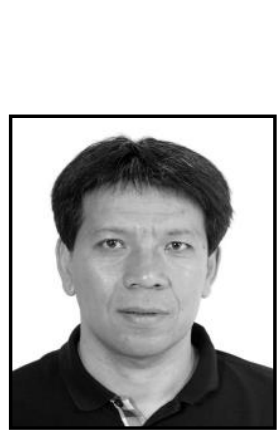

Authors

Gang Wang, received his M.S. degree in Electronic Science from Shenyang Agricultural University, China, in 2011. He is currently a Ph.D. student in College Physics, Dalian University of Technology, China. His main research interests include pattern recognition, new material, and automatic control. Now he is a visiting scholar of Department of Electronic Engineering of Latrobe University, Australia which supported by China Scholarship Council and Shenyang Institute of Engineering.

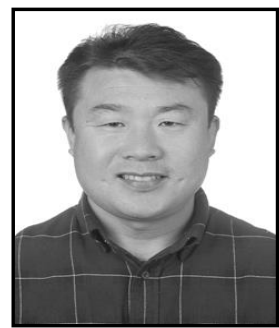

Dan Zhao, received the Master's Degree in operational research and cybernetics from Northeastern Unversity, Shenyang, China, in 2008.

$\mathrm{He}$ is currently a lecture with the Department of Fundamental Teaching, Shenyang Institute of Engineering, Shenyang. His current research interests include sliding mode control, and decentralized control for large-scale systems.

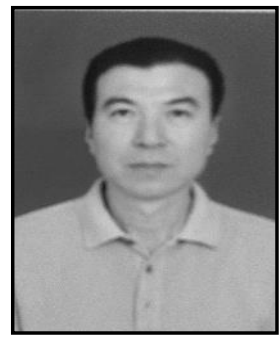

Xiaoyu Sun, he received the MBA degree from Liaoning University, mainly engaged in electrical device with intelligent controlling based on FPGA and soft core CPU. 